\title{
The relationship between transient elastography and histological collagen proportionate area for assessing fibrosis in chronic viral hepatitis
}

\author{
Graziella Isgro • Vincenza Calvaruso • Lorenzo Andreana • Tu Vinh Luong • \\ Matteo Garcovich • Pinelopi Manousou • Angela Alibrandi - Sergio Maimone • \\ Laura Marelli · Neil Davies · David Patch · Amar Paul Dhillon · Andrew Kenneth Burroughs
}

Received: 19 April 2012/ Accepted: 11 September 2012

(C) Springer Japan 2012

\begin{abstract}
Background Collagen proportionate area (CPA) has a better correlation with hepatic venous pressure gradient (HVPG) than with Ishak stage. Liver stiffness measurement (LSM) is proposed as non invasive marker of portal hypertension/disease progression. Our aim was to compare LSM and CPA with Ishak staging in chronic viral hepatitis, and HVPG in HCV hepatitis after transplantation.

Methods One hundred and sixty-nine consecutive patients with chronic hepatitis B virus (HBV) or hepatitis C virus (HCV) infections pre/post liver transplantation (LT), had a liver biopsy combined with LSM (transient elastography), CPA (biopsies stained with Sirius Red and evaluated by digital image analysis and expressed as CPA) and HVPG (measured contemporaneously with transjugular biopsies in LT HCV patients).

Results LSM was dependent on CPA in $\operatorname{HBV}\left(r^{2}=0.61\right.$, $p<0.0001)$, HCV $\left(r^{2}=0.59, p<0.0001\right)$ and LT groups
\end{abstract}

G. Isgro · V. Calvaruso · L. Andreana - M. Garcovich ·

P. Manousou - S. Maimone - L. Marelli · D. Patch ·

A. K. Burroughs $(\bowtie)$

The Royal Free Sheila Sherlock Liver Centre and University Department of Surgery UCL, Royal Free Hospital, Pond Street, Hampstead, London NW3 2QG, UK

e-mail: andrew.burroughs@nhs.net

T. V. Luong · A. P. Dhillon

The Department of Cellular Pathology, UCL Medical School, Royal Free Hospital, London, UK

\author{
A. Alibrandi \\ Department of Economical, Financial, Social, Environmental, \\ Statistical and Territorial Sciences, University of Messina, \\ Messina, Italy \\ N. Davies \\ Department of Radiology, Royal Free Hospital, London, UK
}

$\left(r^{2}=0.64, p<0.0001\right)$. In all three groups, CPA and Ishak were predictors of LSM, but multivariately CPA was better related to LSM (HBV: $r^{2}=0.61, p<0.0001$; HCV: $r^{2}=0.59, p<0.0001$; post-LT: $r^{2}=0.68, p<0.0001$ ) than Ishak stage. In the LT group, multiple regression analysis including HVPG, LSM, aspartate aminotransferase to platelet ratio index (APRI) and Ishak stage/grade, showed that only CPA was related to HVPG $\left(r^{2}=0.41\right.$, $p=0.01)$, both for HVPG $\geq 6 \mathrm{mmHg}$ (OR $1.34,95 \%$ CI $1.14-1.58 ; p<0.0001)$ or $\geq 10 \mathrm{mmHg}(\mathrm{OR} 1.25,95 \% \mathrm{CI}$ $1.06-1.47 ; p=0.007)$.

Conclusion CPA was related to LSM in HBV or HCV hepatitis pre/post-LT. CPA was better related to LSM than Ishak stage. In the LT HCV group, CPA was better related to HVPG than Ishak stage/grade, LSM or APRI. CPA may represent a better comparative histological index for LSM, rather than histological stages.

Keywords Collagen proportionate area - Liver fibrosis . Viral hepatitis · Portal hypertension · Transient elastography $\cdot$ Liver stiffness

\section{Introduction}

Histological assessment of liver fibrosis is the "best standard" for diagnosing and assessing fibrosis in chronic liver disease, in particular for chronic viral hepatitis [1]. The different staging systems, METAVIR scale [2], Ishak [3] and Scheuer [4] systems, assess predominantly architectural changes and do not measure of the degree of liver fibrosis per se. Cirrhosis is only classified as a single stage histologically, being the most severe categorical assignment [5], with no histological sub classification [6]. 
As scoring systems do not quantify fibrosis [5, 6], morphometry is used for this purpose, which could also be used to validate non-invasive markers of liver fibrosis, and to evaluate effects of therapy on fibrosis.

A reliable morphometric method for measuring liver fibrosis is computer-assisted digital image analysis (DIA). The areas of collagen stained with picroSirius red, and of the liver tissue are measured, and the relative proportion of collagen to tissue is derived as the collagen proportionate area (CPA) [7-9].

Calvaruso et al. [9] evaluated CPA, and its relationship with Ishak stage and hepatic venous pressure gradient (HVPG), in patients with recurrent hepatitis $\mathrm{C}$ virus (HCV) after transplantation, finding that CPA was a better histological correlate with HVPG than Ishak stage.

Hepatic venous pressure gradient is used to evaluate portal hypertension in patients with chronic viral hepatitis both pre [10] and post transplantation [11, 12]. Normally HVPG ranges from 1 to $5 \mathrm{mmHg}$; pressures $\geq 6 \mathrm{mmHg}$ indicate portal hypertension (PHT) [13]. HVPG $\geq 10 \mathrm{mmHg}$ predicts the development of complications of cirrhosis [14]. Patients transplanted for HCV cirrhosis with a HVPG $\geq 6 \mathrm{mmHg}$ at 1 year after LT, are at high risk of developing clinical decompensation $[11,12]$. We showed that CPA in 1 year biopsies in patients transplanted for $\mathrm{HCV}$ related cirrhosis was highly predictive of clinical outcome with good sensitivity and specificity, and was better than Ishak stage or HVPG [15]. We also recently demonstrated that CPA increases with worsening cirrhosis in HCV patients post liver transplant, suggesting it can be used for prognostic stratification and assessment of disease progression within the category of severe fibrosis/cirrhosis [16].

To overcome the need to perform liver biopsies to assess the degree of liver fibrosis, a number of non-invasive tests have been developed such as transient elastography (TE) [17-30], and serum direct and indirect markers, such as aspartate aminotransferase (AST) to platelet ratio index (APRI) [31, 32], and recently an algorithm combing 3 biomarkers (3-M-ALG) [34].

The APRI score has been extensively investigated, especially in nontransplant patients with chronic hepatitis C [31, 32]. Only one study [33] evaluated post transplant patients, in which APRI had the highest diagnostic value, compared to other non invasive tests.

Transient elastography gives a liver stiffness measurement (LSM) which correlates with liver disease stage in histological scoring systems. Its performance is best for cirrhosis, but is less accurate in pre-cirrhotic stages [17-21]. LSM correlates better with less severe portal hypertension (HVPG $\leq 10 \mathrm{mmHg}$ ), than with more severe portal hypertension [22-25].

Few studies have been performed using TE in a HCV transplanted patient [26-30]. These show a good correlation between LSM and the liver disease histological stage; one also showed a positive association between LSM and HVPG [29]. LSM may distinguish between slow and rapid "fibrosers" [30].

Morphometric quantitation of collagen has previously been compared with LSM but different stains and methodologies have been used, the correlation between collagen area and LSM was weak and the viral hepatitis groups were not evaluated separately in any of these studies [35-38].

No study exists comparing LSM and CPA in liver transplant patients, and no published study has compared CPA with both LSM and HVPG.

Our aim was to evaluate the relationships between LSM and CPA in patients with chronic viral hepatitis HCV or HBV, and to establish the relationships of both LSM and CPA with Ishak staging, and with HVPG in patients with recurrent $\mathrm{HCV}$ after transplantation.

\section{Methods}

Patients

Between February 2007 and April 2010 we evaluated a consecutive cohort of 169 patients with viral hepatitis who had a liver biopsy, TE and CPA performed concomitantly [9]. The study protocol conformed to the ethical guidelines of the Declaration of Helsinki. We used the specific Ethical permission to examine liver biopsy specimens for research with clinicpathological correlation of histolology with scoring systems, histochemistry and other techniques (when tissue in excess of that needed for diagnostic purposes is available). It was granted by the National Research Ethics Service (REC reference number 07/Q0501/50) with institutional ID number 7558.

All patients gave written informed consent for the procedures and the histological evaluation. The non-transplanted group consisted of 106 consecutive patients with chronic viral hepatitis ( 52 with $\mathrm{HCV}$, and 54 with $\mathrm{HBV}$ ). The transplanted cohort comprised 63 patients transplanted for HCV cirrhosis with a cadaveric graft, who had transjugular liver biopsies (TJB) performed with HVPG $[10,11]$ measured at the same time.

\section{Liver biopsy and hemodynamic study}

The standard percutaneous liver biopsy was performed using the Menghini technique [39], with a 16-gauge disposable needle, in nearly all patients in the HBV and HCV nontransplanted groups. In the remainder TJB was performed.

The TJB and HVPG procedures were performed in all transplanted patients at the time of yearly protocol biopsies or when indicated by abnormal liver function tests for which there was no definitive diagnosis, after $6 \mathrm{~h}$ fast, under local anesthesia (lignocaine 1\%, 5-10 ml 
subcutaneously); $70 \%$ of patients received intravenous sedation with $5-10 \mathrm{mg}$ diazepam. We used a 19-gauge Tru-cut type biopsy needle (Quick core; Cook, William Cook Europe, Denmark) [9] and performed three or four passes through the same hepatic vein wall (right or middle) to ensure that sufficient liver tissue was obtained [40, 41]. Hepatic vein pressures were measured using a 5-Fr balloon catheter using the technique described previously $[10,42]$.

\section{Measurement of CPA}

Liver biopsy samples were formalin fixed, paraffin-embedded and stained with hematoxylin and eosin and Gordon and Sweet staining for reticulin. Another tissue section was stained with picroSirius red for collagen quantification and determination of CPA by DIA. Each biopsy sample was evaluated histologically according to Ishak et al. [2]. For each biopsy, the total length was more than $15 \mathrm{~mm}$ [40]. Portal tracts were defined according to Crawford et al. [43] and were not counted in biopsies with severe distortion of the liver architecture, such as in cirrhosis or marked nodularity, because recognition and enumeration of individual portal tracts in these circumstance is impossible. The section of each biopsy stained with picroSirus red was used for DIA, which was performed by G.I. and V.C. The equipment used consisted of a digital camera (Canon Powershot A 640 attached to a close-up copy-stand with backlighting) connected to a compatible personal computer. After whole section digital image capture, CPA was measured with Zeiss KS300 image analysis software. The CPA measurement included editing steps to eliminate image artifacts and structural collagen in large portal tracts and blood vessel walls as previously described [9]. CPA is expressed as the relative proportion (\%) of collagen to tissue [9].

\section{Transient elastography}

The evaluation was performed before the liver biopsy on the same day, and the HVPG measurement (if performed). Liver stiffness was determined on the right lobe of liver, as previously described [17]. At least ten acquisitions were performed in each patient. The success rate was calculated as the number of validated measurements. The results were expressed in kilopascal $(\mathrm{kPa})$. Only procedures with ten validated measurements and a success rate of at least $60 \%$ and interquartile range (IQR) less than $30 \%$ were considered reliable and used in the analysis.

\section{Statistical analysis}

All data were analyzed using the statistical package SPSS (version 15.0; SPSS Inc, Chicago, IL, USA) and MedCalc statistical software (version 9.3.3). Significance testing was two-sided, and the type 1 error rate was set at 0.05 . Linear regression analysis was used to assess the relationship between LSM and CPA, Ishak stage/grade and the relationship between APRI and CPA, Ishak stage/grade for all patients; HVPG was included in the analysis for transplanted patients only. In the latter group, multiple linear regression analysis was used to evaluate the dependence of HVPG on CPA, LSM, Ishak grade/stage, and APRI score. Logistic regression analysis was used to assess the dependence of the dichotomized HVPG variable (HVPG cut-offs $\geq 6$ and $\geq 10 \mathrm{mmHg}$ representing, respectively, presence of portal hypertension [10], and clinically significant portal hypertension [14]) on CPA, LSM, Ishak stage/grade and APRI. We also evaluated the area under the Receiver Operating Curve (AUROC) for CPA in relation to LSM, in all patients, and also the CPA and LSM in relation to HVPG cut-offs only in transplanted patients. Considering CPA, we divided the range of values in each patient group into quartiles. The cut-offs at 25,50 and $75 \%$ were compared for the three groups of patients, and the median LSM was calculated for each quartile. The statistical significance of inter-quartile differences for LSM was evaluated by the Mann-Whitney test.

As we have previously published [9] and confirmed in this paper, CPA cut-off more than $7 \%$ was the best for distinguishing a normal HVPG from a raised HVPG, and early from advanced stage liver disease, and for this reason we considered patients with CPA $>7 \%$ and $\mathrm{CPA} \leq 7 \%$ separately and evaluated LSM medians and cut-offs for predicting advanced liver fibrosis for each group.

The difference of LSM medians for CPA $>7 \%$ and $\mathrm{CPA} \leq 7 \%$ in each group was evaluated by the MannWhitney test. The difference for LSM medians among the groups for CPA $>7 \%$ and for CPA $\leq 7 \%$ was evaluated by Kruskal-Wallis test.

\section{Results}

One hundred and sixty-nine patient were evaluated in this study: 63 had recurrent HCV hepatitis after liver transplant, 52 chronic HCV hepatitis and 54 chronic HBV hepatitis. Clinical and histological characteristics of the patients are given in Table 1. All patients had a BMI $\leq 30$ and a liver biopsy length $>15 \mathrm{~mm}$.

\section{Relationship between CPA and LSM}

There was a significant dependence of LSM on CPA in all groups: LT group $r^{2}=0.64(\beta=0.80, p<0.0001)$, HCV group $r^{2}=0.59(\beta=0.77, p<0.0001)$ and HBV group $r^{2}=0.61 \quad(\beta=0.78, p<0.0001)$ (Fig. 1). The quartile cut-offs for CPA were very similar in the chronic $\mathrm{HCV}$ 
Table 1 Characteristics of patients with chronic viral hepatitis with respect to evaluation of histological Ishak stage and grade, HVPG [post liver transplant (LT) only], collagen proportionate area (CPA), liver stiffness measurement (LSM), and AST to platelet ratio index (APRI)

\begin{tabular}{llll}
\hline & HCV post LT & HCV & HBV \\
\hline Patients $(n)$ & 63 & 52 & 54 \\
Sex (male) & 49 & 30 & 32 \\
Age (years) & $54(39-66)$ & $51(24-70)$ & $42(24-68)$ \\
BMI $\left(\mathrm{kg} / \mathrm{m}^{2}\right)$ & 23.5 & 24.5 & 24.0 \\
Ishak grade & $4(0-10)$ & $4(2-9)$ & $4(0-10)$ \\
Ishak stage $(n)$ & & & \\
Stage 0-2 & 40 & 30 & 38 \\
Stage 3-4 & 12 & 10 & 10 \\
Stage 5-6 & 11 & 12 & 6 \\
HVPG $(\mathrm{mmHg})$ & $4(0-32)$ & $\mathrm{NP}$ & $\mathrm{NP}$ \\
HVPG $\geq 6 \mathrm{mmHg}$ & 16 & & \\
HVPG $\geq 10 \mathrm{mmHg}$ & 7 & & \\
CPA $(\%)$ & $4.3(0.5-32.0)$ & $4.4(0.5-28.0)$ & $3.0(0.2-24.0)$ \\
LSM $(\mathrm{kPa})$ & $7.2(3.1-34.8)$ & $6.4(3.0-75.0)$ & $6.4(2.8-39.8)$ \\
APRI & $0.9(0.2-19.0)$ & $0.6(0.2-10.5)$ & $0.5(0.2-5.5)$ \\
\hline
\end{tabular}

Results are given in median (ranges)

$N P$ not performed hepatitis and recurrent $\mathrm{HCV}$ hepatitis following liver transplantation groups, whereas these cut-offs were lower in the HBV group.

Considering each quartile of CPA for each group we calculated the median value of LSM, finding a statistically significant difference between the third and the fourth quartile in each group (Table 2).

In the post transplant group, for CPA $>7 \%$ the AUROC for LSM was 0.97 (95\% CI 0.94-1.0) and the best cut-off was $9.0 \mathrm{kPa}(91 \%$ sensitivity and $99 \%$ specificity). The median value of LSM for CPA $\leq 7 \%$ was $6.1 \mathrm{kPa}$ (3.1-19.4), whereas for $\mathrm{CPA}>7 \%$ it was $15.2 \mathrm{kPa}$ (7.4-34.8) $(p<0.0001)$. In the HCV group, for CPA $>7 \%$ the AUROC for LSM was 0.91 (95\% CI 0.81-1.0) and the best cut-off was $9.3 \mathrm{kPa}(94 \%$ sensitivity and $84 \%$ specificity). The median value of LSM for CPA $\leq 7 \%$ was $6.1 \mathrm{kPa}(3.0-14.3)$, whereas for CPA $>7 \%$ it was $14.0 \mathrm{kPa}$ (4.8-75.0) $(p<0.0001)$. Thus the relationships between CPA and LSM are similar for hepatitis C before and after transplantation, despite different widths of biopsy needles used for the standard biopsy and transjugular ones.

In patients with chronic hepatitis $\mathrm{B}$, for $\mathrm{CPA}>7 \%$, AUROC for LSM was 0.99 (95\% CI 0.99-1.0), and the best cut-off of $11.0 \mathrm{kPa}(100 \%$ sensitivity and $98 \%$
Fig. 1 Correlation between CPA and LSM in total group and subgroups: post-LT group, $\mathrm{HCV}$ group and $\mathrm{HBV}$ group
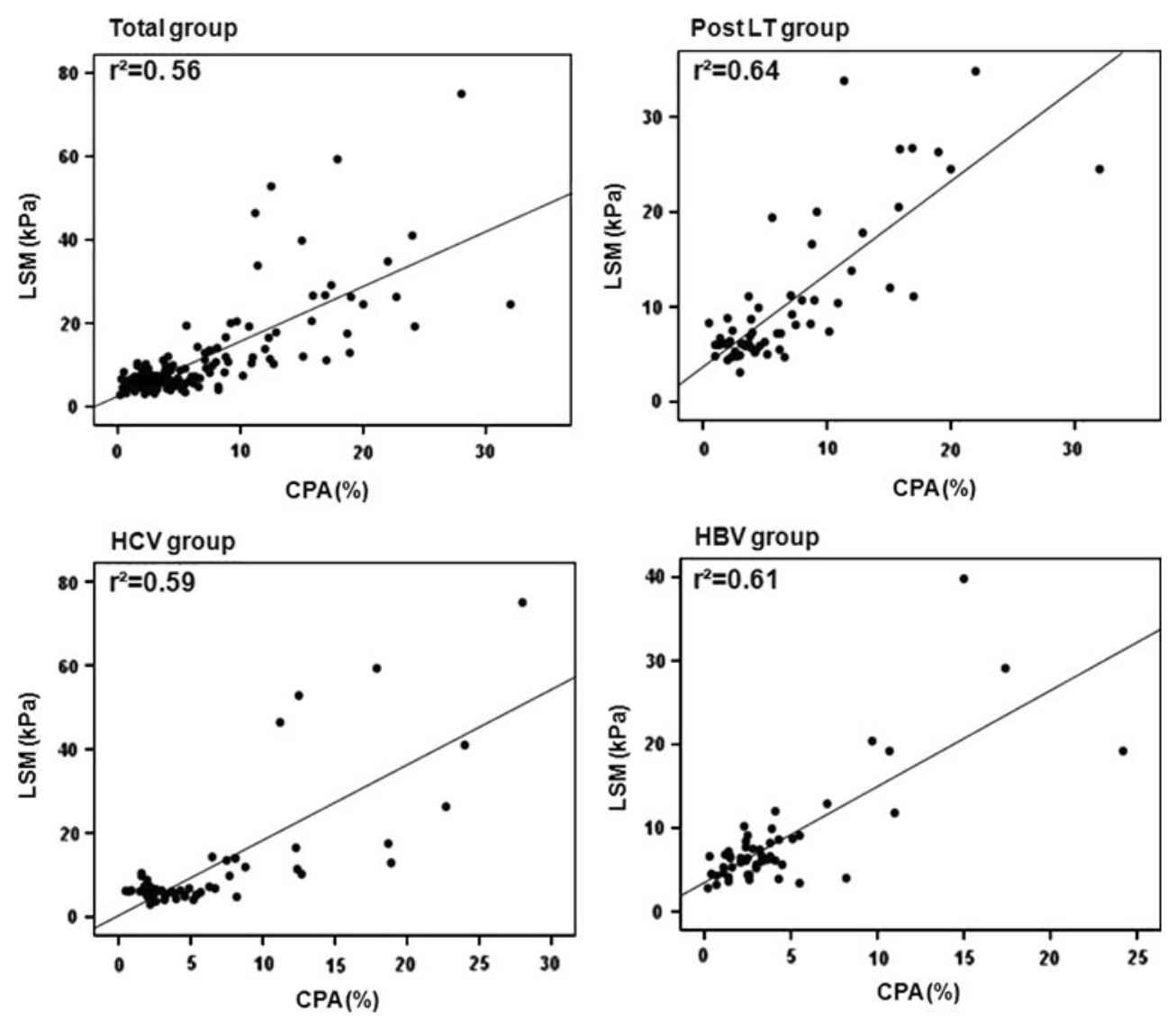
Table 2 Quartiles of CPA values in the 3 groups of patients with chronic viral hepatitis (post transplant HCV, HCV and HBV), and corresponding median LSM values, and Ishak stage scores

\begin{tabular}{|c|c|c|c|c|}
\hline CPA values & 1st quartile $\leq 25 \%$ & 2nd quartile $\leq 50 \%$ & 3rd quartile $\leq 75 \%$ & 4 th quartile $>75 \%$ \\
\hline \multicolumn{5}{|l|}{ Post LT group } \\
\hline Patients $(n)$ & 17 & 15 & 16 & 15 \\
\hline $\mathrm{CPA}(\%)^{\S}$ & $\leq 2.6$ & $\leq 4.3$ & $\leq 9.0$ & $>9.0$ \\
\hline LSM (kPa) & $6.1(4.4-8.8)$ & $5.9(3.1-11.1)^{*}$ & $8.1(4.7-19.4)^{\circ}$ & $20.5(7.4-34.8)^{+}$ \\
\hline Ishak $0-2 / 3-4 / 5 / 6$ & $17 / 0 / 0 / 0$ & $12 / 3 / 0 / 0$ & $10 / 5 / 1 / 0$ & $1 / 4 / 5 / 5$ \\
\hline \multicolumn{5}{|l|}{$\mathrm{HCV}$ group } \\
\hline Patients $(n)$ & 13 & 13 & 13 & 13 \\
\hline $\mathrm{CPA}(\%)$ & $\leq 2.3$ & $\leq 4.4$ & $\leq 8.1$ & $>8.1$ \\
\hline LSM (kPa) & $6.2(3.0-10.4)$ & $5.5(3.7-6.8)^{*}$ & $6.8(4.1-14.3)^{*}$ & $17.5(4.8-75.0)^{++}$ \\
\hline Ishak $0-2 / 3-4 / 5 / 6$ & $13 / 0 / 0 / 0$ & $9 / 3 / 1 / 0$ & $8 / 4 / 0 / 1$ & $0 / 3 / 1 / 9$ \\
\hline \multicolumn{5}{|l|}{ HBV group } \\
\hline Patients $(n)$ & 14 & 16 & 12 & 12 \\
\hline $\mathrm{CPA}(\%)$ & $\leq 1.5$ & $\leq 3.0$ & $\leq 4.3$ & $>4.3$ \\
\hline LSM (kPa) & $4.9(2.8-10.0)$ & $6.0(3.8-10.2)^{*}$ & $6.6(3.4-12.0)^{*}$ & $12.3(3.4-39.8)^{+++}$ \\
\hline Ishak $0-2 / 3-4 / 5 / 6$ & $12 / 2 / 0 / 0$ & $15 / 1 / 0 / 0$ & $8 / 4 / 0 / 0$ & $3 / 3 / 3 / 3$ \\
\hline
\end{tabular}

LSM results are given in median (range)

Differences for LSM between 1st and 2nd, 2nd and 3rd, 3rd and 4th quartiles of CPA values: $* p>0.05,{ }^{\circ} p=0.02,{ }^{+} p<0.0001$, ${ }^{++} p=0.003,{ }^{+++} p=0.05$

$\S \mathrm{CPA}(\%)$ is the relative proportion of collagen to tissue (stained by picroSirius red), called collagen proportionate area

specificity). The median value of LSM for CPA $\leq 7 \%$ was $6.2 \mathrm{kPa}(2.8-12)$, whereas for CPA $>7 \%$ was $19.2 \mathrm{kPa}$ (4-39.8) $(p<0.0001)$.

No significant difference in LSM was found between the three groups for $\mathrm{CPA} \leq 7 \%$ and $\mathrm{CPA}>7 \%$.

Relationship between CPA, LSM and Ishak stage/grade

In all 3 groups, linear regression analysis was used to evaluate the dependence of LSM on CPA, Ishak stage and grade. Univariately LSM was dependent on CPA (post LT: $r^{2}=0.64, \beta=0.80, p<0.0001, \mathrm{HCV}: r^{2}=0.59, \beta=$ $0.80, p<0.0001$, HBV: $\left.r^{2}=0.61, \beta=0.80, p<0.0001\right)$, and Ishak stage (post LT group: $r^{2}=0.60, \beta=0.80$, $p<0.0001$, HCV: $r^{2}=0.37, \beta=0.60, p<0.0001$, HBV: $\left.r^{2}=0.52, \beta=0.70, p<0.0001\right)$. As regards Ishak grade, this was only significant in the post LT group $\left(r^{2}=0.34\right.$, $\beta=0.56, p<0.0001)$. Multivariately CPA was the only independent variable associated with LSM in HCV $\left(r^{2}=0.61, \beta=0.80, p<0.0001\right)$ and HBV $\left(r^{2}=0.69\right.$, $\beta=0.95, p<0.0001)$ groups and it $\left(r^{2}=0.68, \beta=0.47\right.$, $p=0.001)$ was related to LSM better than Ishak stage ( $\beta=0.34, p=0.03)$ in the post LT group. The results confirm the strong relationship of LSM with CPA, which was better than with Ishak stage/grade in each patient group. The scatter plots for the relation between CPA and LSM, and Ishak are shown in Figs. 1 and 2, respectively; for LSM and Ishak in Fig. 3. It should be noted that Ishak stages described as numbers do not have a quantitative relationship between them as we have discussed previously [3-7], and this may account for the better relationship seen between LSM and CPA, compared to relationships with the Ishak stage.

Relationship between CPA and APRI score

Collagen proportionate area was significantly related to APRI in all three groups. The evaluation of APRI and CPA, Ishak stage and grade showed that univariately APRI was significantly dependent on CPA (post LT: $r^{2}=0.11$, $\beta=0.37, p=0.01 ; \mathrm{HCV}: r^{2}=0.38, \beta=0.62, p<$ 0.0001 , HBV: $\left.r^{2}=0.19, \beta=0.43, p=0.002\right)$ and Ishak stage (post LT: $r^{2}=0.14, \beta=0.37, p=0.004$; HCV: $r^{2}=0.23, \quad \beta=0.47, \quad p<0.0001 ; \quad$ HBV: $r^{2}=0.23$, $\beta=0.48, p=0.001)$, but it was dependent on Ishak grade in the HBV group only ( $r^{2}=0.14, \beta=0.37, p=0.016$ ). In the multiple regression analysis, CPA was independently associated with APRI score only in the HCV group $\left(r^{2}=0.40, \beta=0.68, p=0.007\right)$.

Relationships between LSM, CPA, APRI score and Ishak stage/grade with HVPG in the transplanted patients with recurrent $\mathrm{HCV}$

In the 63 patients post LT, univariately HVPG was dependent on CPA ( $\left.r^{2}=0.4, \beta=0.47, p=0.000\right)$, Ishak 
Fig. 2 Correlation between CPA and Ishak stage in total group and subgroups: post-LT group, HCV group and HBV group

Fig. 3 Correlation between LSM and Ishak stage in total group and subgroups: post-LT group, HCV group and HBV group
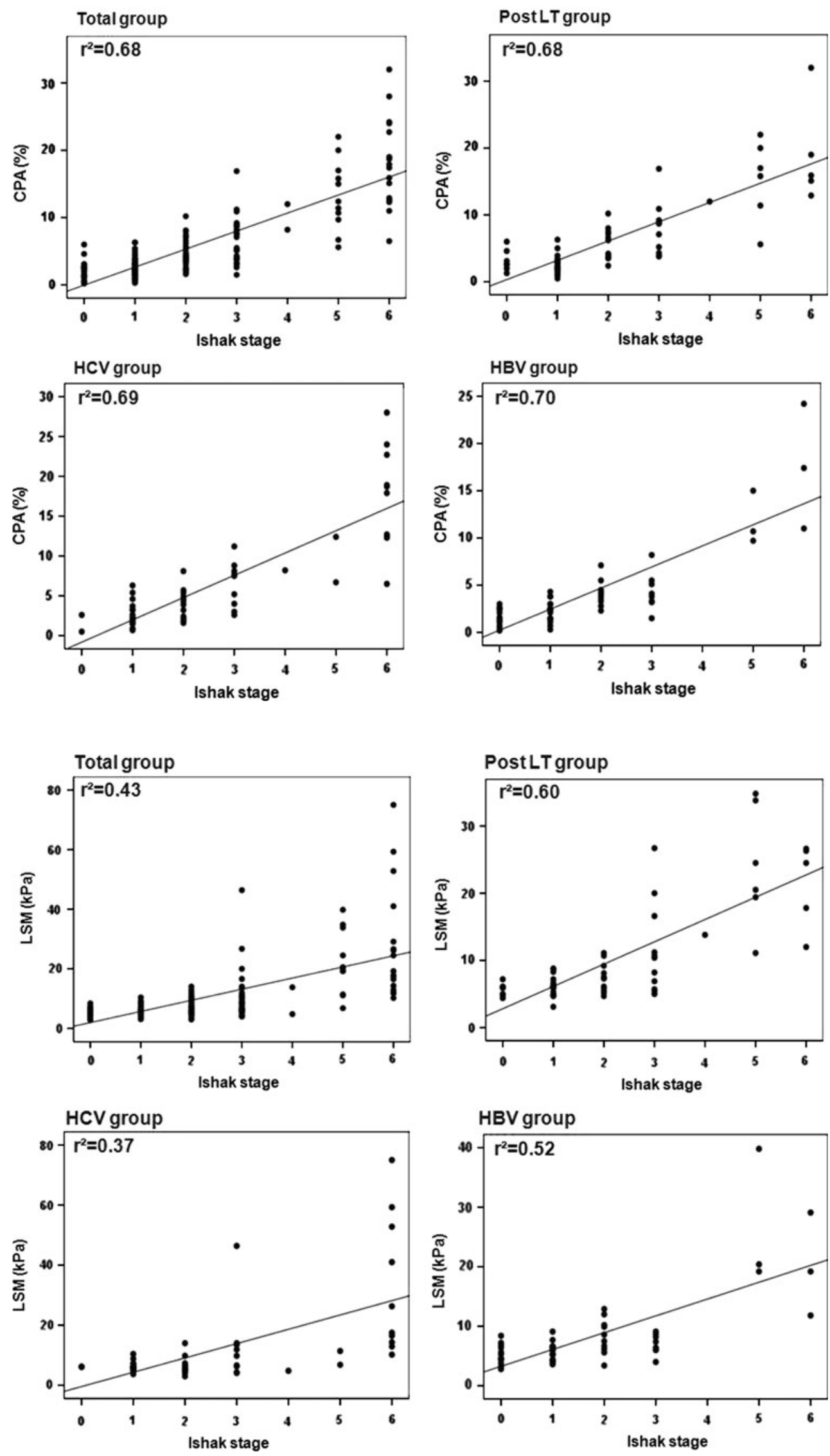
stage $\left(r^{2}=0.35, \beta=0.60, p=0.0001\right)$, Ishak grade $\left(r^{2}=0.11, \quad \beta=0.33, \quad p=0.012\right), \quad$ LSM $\quad\left(r^{2}=0.22\right.$, $\beta=0.50, p=0.000)$, and APRI $\left(r^{2}=0.08, \beta=0.29\right.$, $p=0.029$ ). However, in a multivariate analysis, CPA remained the only feature influencing HVPG $\left(r^{2}=0.41\right.$, $\beta=0.56, p=0.01$ ).

\section{Patients with portal hypertension ( $H V P G \geq 6 \mathrm{mmHg}$ )}

There were 16 patients. Portal hypertension was related to CPA $(p<0.0001)$, LSM $(p<0.0001)$, Ishak stage $(p<0.0001)$, Ishak grade $(p=0.023)$ and APRI score $(p=0.04)$. Multivariately only CPA (OR $1.34,95 \%$ CI $1.14-1.6, p<0.0001)$ remained significantly associated with $\mathrm{HVPG} \geq 6 \mathrm{mmHg}$.

For HVPG $\geq 6 \mathrm{mmHg}$, the AUROC for CPA was 0.91 (95\% CI 0.83-0.98). The best cut-off was $7.7 \%$ (75\% sensitivity and $89 \%$ specificity); AUROC for LSM was 0.83 (95\% CI 0.70-0.95), and the cut-off of $10.9 \mathrm{kPa}$ had $75 \%$ sensitivity and $89 \%$ specificity. Thus, for the presence of portal hypertension, CPA had a better association than LSM (Fig. 4).

\section{Patients with significant portal hypertension $(H V P G \geq 10 \mathrm{mmHg}$ )}

There were seven patients. In the univariate analysis we found a significant dependence on CPA $(p<0.0001)$, LSM $(p=0.001)$ Ishak stage $(p=0.005)$, Ishak grade $(p=$ $0.013)$ APRI score $(p=0.002)$. Multivariately only CPA

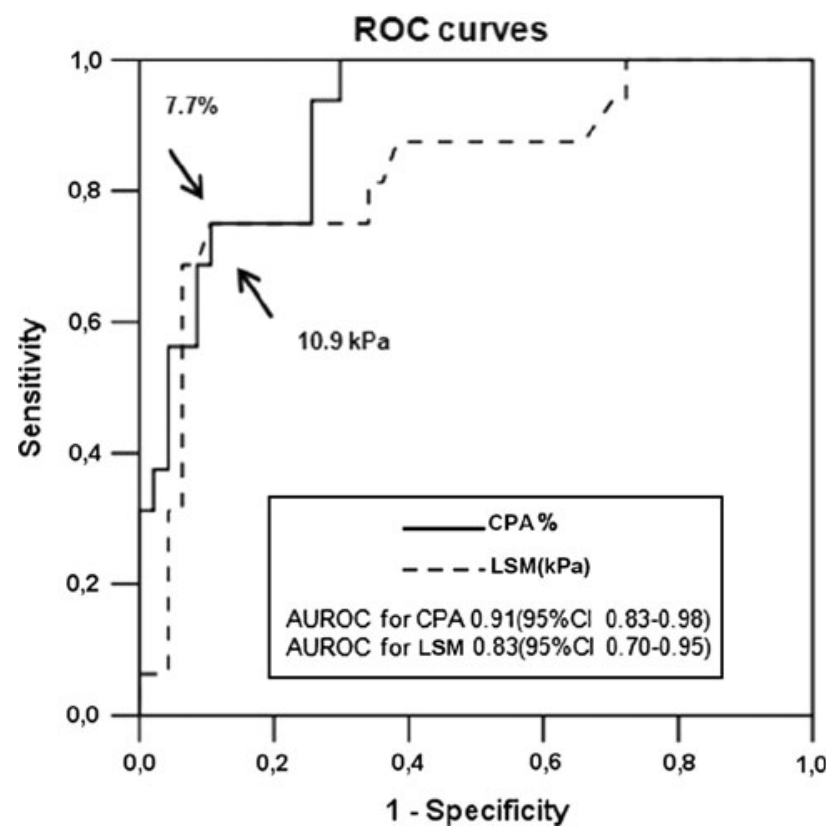

Fig. 4 ROC curves for LSM and CPA for 16 patients with portal hypertension (HVPG $\geq 6 \mathrm{mmHg}$ ) post-LT
(OR $1.25,95 \%$ CI $1.06-1.47, p=0.007)$ was significantly associated with $\mathrm{HVPG} \geq 10 \mathrm{mmHg}$.

\section{Discussion}

We assessed CPA and compared this with LSM in three groups of patients with chronic viral hepatitis: 54 with chronic hepatitis B, 52 with chronic hepatitis $\mathrm{C}$ and 63 transplanted patients with recurrent HCV. In transplanted patients we also obtained HVPG. The LSM and APRI score calculations were performed at the same time as obtaining a three or four core TJB or percutaneous biopsy [40, 41]. Liver histology was scored according to Ishak et al. [3], and we used our previously published methods for assessing hepatic collagen content using DIA and evaluating CPA [9]. In each patient group, CPA correlated well with LSM. Moreover, in each group the relationship between CPA and LSM was better than the relationship between Ishak stage and LSM. Most previous publications have used the histological stage to compare with LSM [1721]. Our data on CPA, which is a continuous quantitative variable, suggests this is a better histological index than the categorical stage scores, to compare with LSM and it is likely that this could be the case for all non-invasive tests.

The results support our view that using categorical descriptive variables (grade and stage scores) as a quantitative measure of fibrosis is not only incorrect methodologically $[5,6]$, but is also a poor "best" standard to compare to LSM [44].

Dividing the CPA values in each group into quartiles, the transplanted $\mathrm{HCV}$ and non-transplanted HCV groups had similar values, whereas the CPA values for HBV group were lower, probably related to fewer patients with cirrhosis in this group. However, in this study was not possible to confirm this. The LSM medians between the adjacent quartiles of CPA in each patient group showed a statistically significant difference between the third and fourth quartile. This suggests that LSM has a better association with higher degrees of fibrosis. Indeed LSM is better at identifying higher disease stages (cirrhosis) rather than earlier stages of disease [17-21]. Using the AUROC cut-off of CPA $>7 \%$ associated with $\mathrm{HVPG} \geq 6 \mathrm{mmHg}$ (portal hypertension), the LSM cut-offs for HCV with or without transplantation were also similar ( 9.0 and $9.3 \mathrm{kPa})$. In the post transplant group, we found as before [9] that HVPG was related to both stage and LSM, as in other studies [22-24]. However when CPA was considered with LSM, Ishak stage, grade APRI score and HVPG all together, then multivariately only CPA was independently related to HVPG. As HVPG is a robust prognostic marker [13, 45], this suggests CPA is likely to be a robust prognostic histological marker [15]. The CPA was also the only 
variable independently related to $\mathrm{HVPG} \geq 6 \mathrm{mmHg}$ and HVPG $\geq 10 \mathrm{mmHg}$. Thus, CPA has a good relationship with HVPG across a wide range of values, better than LSM. It is important to note that there were only 7 of 63 patients with HVPG $\geq 10 \mathrm{mmHg}$, so that LSM was assessed in the HVPG range where LSM is known to have a better correlation with HVPG [22].

Our results are not the only ones assessing collagen morphometrically, but due to different methodologies used in other publications [35-38], a direct comparison is not possible. However, our data are similar to others who have evaluated chronic hepatitis, but in several papers, different etiologies are bunched together. For example Ziol et al. [38] evaluated HCV and HBV patients together. This also makes direct comparisons with our study difficult.

In conclusion we have shown that firstly, CPA is a good measure to quantify fibrosis in liver biopsies of nontransplanted patients with viral hepatitis $\mathrm{C}$ and $\mathrm{B}$ and recurrent $\mathrm{HCV}$ similar to our previous finding in transplanted patients with recurrent HCV [9]. Secondly, CPA in recurrent chronic viral hepatitis $\mathrm{C}$ after liver transplantation [9], has a similar relationship to LSM, as in non-transplant chronic hepatitis C patients; moreover, in recurrent $\mathrm{HCV}$ hepatitis, CPA has a better relationship with HVPG than Ishak stage, LSM or APRI. Thirdly, the relationship between LSM and CPA was better than the relationship between LSM and Ishak stages in all chronic viral hepatitis groups. Our data suggest that CPA should be the histological parameter with which to compare LSM and other non-invasive markers as we have suggested previously [5, $6,9]$. It should also be used to sub-classify cirrhosis histologically $[9,46]$.

Acknowledgments No specific funding was available other than salaries from Royal Free Hospital Hampstead, NSH Trust and UCL Medical School.

Conflict of interest The authors declare that they have no conflict of interest.

\section{References}

1. Bedossa P. Harmony in liver fibrosis. J Hepatol. 2010;52:313-4.

2. Poynard T, Bedossa P, Opolon P. Natural history of liver fibrosis progression in patients with chronic hepatitis C. The OBSVIRC, METAVIR, CLINIVIR and DOSVIRC groups. Lancet. 1997; 349:825-32.

3. Ishak K, Baptista A, Bianchi L, Callea F, De Groote J, Gudat F, et al. Histological grading and staging of chronic hepatitis. J Hepatol. 1995;22:696-9.

4. Knodell RG, Ishak KG, Black WC, Chen TS, Craig R, Kaplowitz $\mathrm{N}$, et al. Formulation and application of a numerical scoring system for assessing histological activity in asymptomatic chronic active hepatitis. Hepatology. 1981;1:431-5.
5. Standish RA, Cholongitas E, Dhillon A, Burroughs AK, Dhillon AP. An appraisal of histopathological assessment of liver fibrosis. Gut. 2006;55:569-78.

6. Germani G, Burroughs AK, Dhillon AP. The relationship between liver disease stage and liver fibrosis: a tangled web. Histopathology. 2010;57:773-4.

7. Goodman ZD, Becker RL, Pockros PJ, Afdhal NH. Progression of fibrosis in advanced chronic hepatitis $\mathrm{C}$ : evaluation by morphometric image analysis. Hepatology. 2007;45:886-94.

8. Fontana RJ, Goodman ZD, Dienstag JL, Bonkovsky HL, Naishadham D, Sterling RK, et al. Relationship of serum fibrosis markers with liver fibrosis stage and collagen content in patients with advanced chronic hepatitis C. Hepatology. 2008;47:1-10.

9. Calvaruso V, Burroughs AK, Standish R, Manousou P, Grillo F, Leandro $\mathrm{G}$, et al. Computer-assisted image analysis of liver collagen: relationship to Ishak scoring and hepatic venous pressure gradient. Hepatology. 2009;49:1236-44.

10. Burroughs AK, Groszmann RJ, Bosch J, Grace N, Garcia-Tsao G, Patch D, et al. Assessment of therapeutic benefit of antiviral therapy in chronic hepatitis $\mathrm{C}$ : is hepatic venous pressure gradient a better end point? Gut. 2002;50:425-7.

11. Samonakis D, Cholongitas E, Thalheimer U, Kalambokis G, Quaglia A, Triantos C, et al. Hepatic venous pressure gradient to assess fibrosis and its progression after liver transplantation for HCV cirrhosis. Liver Transpl. 2007;13:1305-11.

12. Blasco A, Forns X, Carrion JA, Garcia-Pagan JC, Gilabert R, Rimola A, et al. Hepatic venous pressure gradient identifies patients at risk of severe hepatitis $\mathrm{C}$ recurrence after liver transplantation. Hepatology. 2006;43:492-9.

13. Burroughs AK, Thalheimer U. Hepatic venous pressure gradient in 2010: optimal measurement is key. Hepatology. 2010;51: 1894-6.

14. Ripoll C, Groszmann R, Garcia-Tsao G, Bosh J, Grace N, Burroughs A, et al. Hepatic venous pressure gradient predicts clinical decompensation in patients with compensated cirrhosis. Gastroenterology. 2007;133:481-8.

15. Manousou P, Dhillon AP, Isgro G, Calvaruso V, Luong TV, Tsochatzis E, et al. Computer-Assisted Image Analysis of Liver Collagen at one year biopsy can predict clinical outcome in HCV post LT patients. Liver Transpl. 2011;17:178-88.

16. Calvaruso V, Dhillon AP, Tsochatzis E, Manousou P, Grillo F, Germani G, et al. Histological Liver Collagen Proportionate Area predicts decompensation in patient with severe fibrosis due to $\mathrm{HCV}$ recurrence after liver transplantation. J Gastroenterol Hepatol. 2012;27:1227-32.

17. Sandrin L, Fourquet B, Hasquenoph JM, Yon S, Fournier C, Mal F, et al. Transient elastography: a new noninvasive method for assessment of hepatic fibrosis. Ultrasound Med Biol. 2003;29: 1705-13.

18. Ziol M, Handra-Luca A, Kettaneh A, Christidis C, Mal F, Kazemi $\mathrm{F}$, et al. Noninvasive assessment of liver fibrosis by measurement of stiffness in patients with chronic hepatitis C. Hepatology. 2005;41:48-54.

19. Marcellin P, Ziol M, Bedossa P, Douvin C, Poupon R, de Lédinghen $\mathrm{V}$, et al. Non-invasive assessment of liver fibrosis by stiffness measurement in patients with chronic hepatitis B. Liver Int. 2009;29:242-7.

20. Foucher J, Chanteloup E, Vergniol J, Castéra L, Le Bail B, Adhoute X, et al. Diagnosis of cirrhosis by transient elastography (FibroScan): a prospective study. Gut. 2006;55:403-8.

21. Tsochatzis EA, Gurusamy KS, Ntaoula S, Cholongitas E, Davidson BR, Burroughs AK. Elastography for the diagnosis of severity of fibrosis in chronic liver disease: a meta-analysis of diagnostic accuracy. J Hepatol. 2011;54:650-9.

22. Vizzutti F, Arena U, Romanelli RG, Rega L, Foschi M, Colagrande S, et al. Liver stiffness measurement predicts severe portal 
hypertension in patients with HCV-related cirrhosis. Hepatology. 2007;45:1290-7.

23. Lemoine M, Katsahian S, Ziol M, Nahon P, Ganne-Carrie N, Kazemi F, et al. Liver stiffness measurement as a predictive tool of clinically significant portal hypertension in patients with compensated hepatitis $\mathrm{C}$ virus or alcohol-related cirrhosis. Aliment Pharmacol Ther. 2008;28:1102-10.

24. Bureau C, Metivier S, Peron JM, Selves J, Robic MA, Gourraud PA, et al. Transient elastography accurately predicts presence of significant portal hypertension in patients with chronic liver disease. Aliment Pharmacol Ther. 2008;27:1261-8.

25. Bosh J. Soft technique, hard end-points. J Hepatol. 2011;55: 955-6.

26. Corradi F, Piscaglia F, Flori S, D'Errico-Grigioni A, Vasuri F, Tame MR, et al. Assessment of liver fibrosis in transplant recipients with recurrent $\mathrm{HCV}$ infection: usefulness of transient elastography. Dig Liver Dis. 2009;41:217-25.

27. Rigamonti C, Donato MF, Fraquelli M, Agnelli F, Ronchi G, Casazza G, et al. Transient elastography predicts fibrosis progression in patients with recurrent hepatitis $\mathrm{C}$ after liver transplantation. Gut. 2008;57:821-7.

28. Sanchez Antolin G, Garcia Pajares F, Vallecillo MA, Fernandez Orcajo P, Gomez de la Cuesta S, Alcaide N, et al. FibroScan evaluation of liver fibrosis in liver transplantation. Transplant Proc. 2009;41:1044-6.

29. Carrion JA, Navasa M, Bosch J, Bruguera M, Gilabert R, Forns $\mathrm{X}$. Transient elastography for diagnosis of advanced fibrosis and portal hypertension in patients with hepatitis $\mathrm{C}$ recurrence after liver transplantation. Liver Transpl. 2006;12:1791-8.

30. Carrion JA, Navasa M, Bosch J, Bruguera M, Gilabert R, Forns $X$. Liver stiffness identifies two different patterns of fibrosis progression in patients with hepatitis $\mathrm{C}$ virus recurrence after liver transplantation. Hepatology. 2010;51:23-34.

31. Burroughs AK, Cholangitas E. Non-invasive tests for liver fibrosis: encouraging or discouraging results? J Hepatol. 2007;46: $751-5$.

32. Pohl A, Behling C, Oliver D, Kilani M, Monson P, Hassanein T. Serum aminotransferase levels and platelet counts as predictors of degree of fibrosis in chronic hepatitis $\mathrm{C}$ virus infection. Am J Gastroenterol. 2001;96:3142-6.

33. Toniutto P, Fabris C, Bitetto D, Falleti E, Avellini C, Rossi E, et al. Role of AST to platelet ratio index in the detection of liver fibrosis in patients with recurrent hepatitis $\mathrm{C}$ after liver transplantation. J Gastroenterol Hepatol. 2007;22:1904-8.

34. Carrión JA, Fernández-Varo G, Bruguera M, García-Pagán JC, García-Valdecasas JC, Pérez-Del-Pulgar S, et al. Serum fibrosis markers identify patients with mild and progressive hepatitis C recurrence after liver transplantation. Gastroenterology. 2010;138: $147-58$.

35. Kawamoto M, Mizuguchi T, Katsuramaki T, Nagayama M, Oshima H, Kawasaki $\mathrm{H}$, et al. Assessment of liver fibrosis by noninvasive method of transient elastography and biochemical markers. World J Gastroenterol. 2006;12:4325-30.

36. Nitta Y, Kawabe N, Hashimoto S, Harata M, Komura N, Kobayashi K, et al. Liver stiffness measured by transient elastography correlates with fibrosis area in liver biopsy in patients with chronic hepatitis C. Hepatol Res. 2009;39:675-84.

37. Wong GL, Wong VW, Choi PC, Chan AW, Chum RH, Chan HK, et al. Assessment of fibrosis by transient elastography compared with liver biopsy and morphometry in chronic liver diseases. Clin Gastroenterol Hepatol. 2008;6:1027-35.

38. Ziol M, Kettaneh A, Ganne-Carrie N, Barget N, Tengher-Barna I, Beaugrand M. Relationships between fibrosis amounts assessed by morphometry and liver stiffness measurements in chronic hepatitis or steatohepatitis. Eur J Gastroenterol Hepatol. 2009;21: 1261-8.

39. Menghini G. One-second biopsy of the liver-problems of its clinical application. N Engl J Med. 1970;283:582-5.

40. Shusang V, Marelli L, Cholongitas E, Kalambokis G, Manousou $\mathrm{P}$, Senzolo M, et al. Transjugular liver biopsy: are 4 cores better than 3 and does a cassette prevent fragmentation? J Hepatol. 2007;46(Suppl 1):S76.

41. Cholongitas E, Quaglia A, Samonakis D, Senzolo M, Triantos C, Patch D, et al. Transjugular liver biopsy: how good is it for accurate histological interpretation? Gut. 2006;55:1789-94.

42. Groszmann RJ, Wongcharatrawee S. The hepatic venous pressure gradient: anything worth doing should be done right. Hepatology. 2004;39:280-2.

43. Crawford AR, Lin XZ, Crawford JM. The normal adult human liver biopsy: a quantitative reference standard. Hepatology. 1998; 28:323-31.

44. Garcia-Tsao G, Friedman S, Iredale J, Pinzani M. Now there are many (stages) where before there was one: in search of a pathophysiological classification of cirrhosis. Hepatology. 2010;51: 1445-9.

45. Trinatos CK, Nikolopoulou V, Burroughs AK. Review article: the therapeutic and prognostic benefit of portal pressure reduction in cirrhosis. Aliment Pharmacol Ther. 2008;28:943-52.

46. Germani G, Dhillon A, Andreana L, Calvaruso V, Manousou P, Isgro $\mathrm{G}$, et al. Histological subclassification of cirrhosis. Hepatology. 2010;52:804-5. 